\title{
Elektrolyseure für eine nachhaltige Energiewirtschaft
}

\author{
Die Universität Bayreuth erforscht im $\mathrm{H}_{2}$ Giga-Projekt die Entwicklung und industrielle \\ Fertigung leistungsstarker und kostengünstiger Elektrolyseure, mit denen der Bedarf \\ Deutschlands an grünem Wasserstoff künftig gedeckt werden kann.
}

Grüner Wasserstoff, der große Mengen nachhaltig erzeugter Energie speichert und über lange Strecken transportiert werden kann, ist von zentraler Bedeutung für die künftige Energieversorgung. Schon heute ist absehbar, dass sich der künftige Bedarf allein in Deutschland auf mehrere hundert Millionen Tonnen jährlich belaufen wird. Zur Deckung dieses Bedarfs sind effiziente, robuste und kostengünstige Elektrolyseure erforderlich, die mittels elektrischer Energie aus nachhaltigen Quellen Wassermoleküle aufspalten und so Wasserstoff erzeugen. Die Elektrolyseure müssen in industrieller Serienfertigung hergestellt werden und in der Lage sein, bis 2030 die von der Wasserstoffstrategie der Europäischen Union vorgegebene Zielmarke von 40 Gigawatt Elektrolysekapazität zu erreichen.

Die Hochtemperaturelektrolyse (HTEL) hat sich als eine besonders vielversprechende Technologie zur Erzeugung von grünem Wasserstoff erwiesen. Als Elektrolyseure dienen hintereinander geschaltete HTEL-Zellen, die als HTEL-Stacks bezeichnet werden.

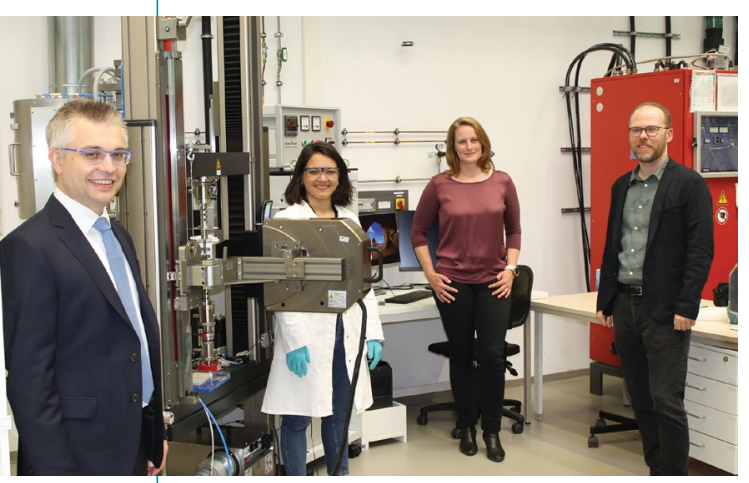

Gemeinsame Forschung zur Hochtemperaturelektrolyse am Lehrstuhl Keramische Werkstoffe: Prof. Dr.-Ing. Stefan Schafföner, Ilaria Bombarda M.Sc., Dr.-Ing. Carolin Sitzmann und Dr. rer. nat. Nico Langhof (v.l.n.r.). (๑ UBT / Christian Wißler)
Damit der Energiewirtschaft in Zukunft großskalige HTEL-Zellen und HTEL-Stacks zur Verfügung stehen, sind allerdings noch erhebliche Forschungs- und Entwicklungsschritte nötig: Sie betreffen die Lebensdauer, die Materialkosten, die Effizienz, neue Technologien zur Fertigung der Stacks sowie deren Einsatz für die Wasserstoffproduktion in den benötigten hohen Mengen.

\section{Alterungsprozesse vorhersagen}

Genau hier setzt das $\mathrm{H}_{2}$ Giga-Projekt „HTs: HTEL-Stacks - Ready for Gigawatt“ an. Der Lehrstuhl Keramische Werkstoffe an der Universität Bayreuth ist hier für entscheidende Forschungs- und Entwicklungsschritte verantwortlich: Sowohl neue als auch schon im Betrieb befindliche Elektrolyseurzellen, die auf einem Elektrolyten aus Zirkonoxid basieren, sollen auf ihre Mikrostruktur und thermomechanischen Eigenschaften hin untersucht werden. Dabei ist es besonders wichtig, dass die Festigkeit der Zellen bei hohen Temperaturen bis zu $850^{\circ} \mathrm{C}$ erhalten bleibt. Nur wenn die Zusammenhänge zwischen der Mikrostruktur und den thermomechanischen Eigenschaften wissenschaftlich verstanden sind, wird es möglich sein, Alterungsprozesse in den Zellen vorherzusagen und Strategien für eine hohe Langlebigkeit zu entwickeln. „Mit den speziellen Kompetenzen und langjährigen Forschungserfahrungen, die wir in früheren Projekten zur Brennstoffzelle und zur Charakterisierung von sehr dünnen keramischen Folien gewonnen haben, werden wir von Bayreuth aus wichtige Beiträge zu einer nachhaltigen Energiewirtschaft auf der Basis von Wasserstoff leisten können", sagt Prof. Dr.-Ing. Stefan Schafföner, Inhaber des Lehr- stuhls Keramische Werkstoffe. Die Forschungsarbeiten seines Teams werden rückwirkend ab dem 1. Mai 2021 bis zum 31. März 2025 gefördert.

\section{Einzigartige Prüfanlage im Einsatz}

Bei den anstehenden Arbeiten in Bayreuth werden experimentelle Forschungsmethoden zum Einsatz kommen, wie beispielsweise die Licht- und Rasterelektronenmikroskopie, die Röntgenbeugung und die zerstörungsfreie Impulserregungstechnik. Mechanische Kennwerte an den keramischen dünnen Schichten werden dabei bei bis zu $850{ }^{\circ} \mathrm{C}$ durch Doppelring-Biegeversuche sowie Zugversuche unter Verwendung des Laserextensometers ermittelt. Hierfür wird die neue, einzigartige HochtemperaturPrüfanlage des Lehrstuhls eingesetzt, die Ende 2020 in Betrieb ging und durch Mittel der Deutschen Forschungsgemeinschaft (DFG) sowie der TAO (Technologie Allianz Oberfranken) finanziert wurde.

Neben den experimentellen Arbeiten werden auch Simulationen mithilfe der Finite-Elemente-Methode zur Analyse der Lebensdauer durchgeführt. Vor allem bei Fragen zur industriellen Umsetzung der HTEL-Stacks arbeitet der Lehrstuhl Keramische Werkstoffe mit zahlreichen Unternehmen aus Industrie und Forschung zusammen, die ebenfalls am $\mathrm{H}_{2}$ Giga-Projekt beteiligt sind. Die organisatorische Gesamtleitung liegt bei dem Unternehmen Sunfire in Dresden.

Kontakt:

Universität Bayreuth,

Lehrstuhl Keramische Werkstoffe, www.cme-keramik.uni-bayreuth.de 